\title{
Active Flow Control of a Low Reynolds Number S809 Wind Turbine Blade Model under Dynamic Pitching Maneuvers
}

\author{
Victor Maldonado ${ }^{1}$, Soham Gupta ${ }^{2}$ \\ ${ }^{1}$ Department of Mechanical Engineering, University of Texas at San Antonio, San Antonio, TX, USA \\ ${ }^{2}$ Department of Aerospace Engineering and Mechanics, University of Alabama, Tuscaloosa, AL, USA \\ Email: victor.maldonado@utsa.edu,soham12003@gmail.com
}

How to cite this paper: Maldonado, V. and Gupta, S. (2017) Active Flow Control of a Low Reynolds Number S809 Wind Turbine Blade Model under Dynamic Pitching Maneuvers. Open Journal of Fluid Dynamics, 7, 178-193.

https://doi.org/10.4236/ojfd.2017.72012

Received: March 24, 2017

Accepted: June 6, 2017

Published: June 9, 2017

Copyright ( 2017 by authors and Scientific Research Publishing Inc. This work is licensed under the Creative Commons Attribution International License (CC BY 4.0).

http://creativecommons.org/licenses/by/4.0/

\begin{abstract}
A low Reynolds number wind turbine blade model based on the S809 airfoil was tested in a subsonic wind tunnel to study the structural vibration of the blade under dynamic pitching maneuvers. Piezoelectric-based synthetic jet actuators were embedded inside the blade and activated with a synthetic jet momentum coefficient, $C_{\mu}$ of $2.30 \times 10^{-3}$. Structural vibration was quantified for a range of unsteady angles undergoing "pitch up and down" and "sinusoidal pitch" maneuvers at a Reynolds number of $5.28 \times 10^{4}$. The blade tip deflection amplitude and frequency were acquired utilizing a pair of strain gauges mounted at the root of the model. Using active flow control vibration reduction was more effective during the pitch up portion of the blade motion cycle compared to the pitch down portion. This effect is due to dynamic stall, where a leading edge vortex is shed during the pitch up motion and contributes to higher lift compared to static angles of attack and lower lift when the blade is pitched down. Dynamic stall was measured with phase-locked stereoscopic particle image velocimetry (SPIV), where global mean flow measurements reveal a shift in location and reduction in the size of a recirculating flow structure near the suction surface of the blade during the pitch up motion compared to the pitch down.
\end{abstract}

\section{Keywords}

Active Flow Control, Structural Vibration, Dynamic Stall

\section{Introduction}

As wind turbine blades become larger, there becomes an increasing interest to develop active methods to regulate blade loads, and consequently, reduce structural vibration that directly impacts the performance and life of the turbine. 
There are primarily two control methods to keep blade loads within acceptable limits. Stall regulation maintains the blade pitch fixed and operates the turbine at a near constant rotational speed. As the wind speed increases, the angle of attack increases causing the blades to eventually stall resulting in a decrease in lift and an increase in drag, yielding a lower tangential load. The other more effective method to reduce blade loads utilized in modern wind turbines is variable rotational speed and individual blade pitch control, and whereby a large actuator mechanism is connected to each blade and controlled independently. In addition to regulating blade loads, individual blade control is principally used to limit output power and torque in above-rated wind speeds in order to keep turbine operation within its design limits. While this type of control method has the ability to respond sufficiently to changing wind speeds (due to blade rotation) and thus asymmetric aerodynamic loading at each blade at a relatively slow once-per-revolution manner, it cannot respond to higher frequency atmospheric phenomena such as turbulence, wind gusts, and wind shear. Gusts can result in a sudden increase in the blade effective angle of attack, which can yield high unsteady loads on the blade leading to structural failures. These loads are often unavoidable due to the slow response of conventional pitch control actuators in decreasing the angle of attack; hence the development of locally distributed high bandwidth flow control actuators with built in intelligence embedded on the blades is needed.

In recent years, there have been numerous studies on the application of active flow control with piezo-bimorph actuators and/or MEMS (Micro Electrical Mechanical Systems) for active performance enhancement, including load and vibration reduction of wind turbine blades. Most of these studies aim at the feasibility of manipulating aerodynamic loads using such actuators attached to control surfaces (typically near the trailing edge of the blade) commonly referred to as flaps, micro tabs, etc. The use of micro tabs as aerodynamic devices for load control on wind turbine blades has been extensively investigated by van Dam [1] [2] [3] [4], where active micro tabs deployed near the trailing edge on the suction surface of a blade have the ability to reduce the lift and therefore loads on the blade. Wingerden et al. [5] showed a proof of concept study of a 'smart' 3-D rotor blade with trailing edge flaps and a feedback controller for load control. Research on this topic has also been performed in wind industry where trailing edge flaps [6]-[12] and MEMS tabs [13] have been used for load alleviation including their feasibility on a rotating blade, Buhl et al. [12].

The field of active flow control in general (including the use of piezoelectric driven synthetic jets and blowing and suction mechanisms) is not new, and has generated an extensive amount of research findings where most of the focus has been largely on control of separation. Separation control using synthetic jets has recently been demonstrated as a viable means to reduce wind turbine blade structural vibration, Maldonado et al. [14] by exploiting the narrow-band receptivity of the separating shear layer and the upstream boundary layer to external actuation. Previously, Oster and Wygnanski [15] and Roberts [16] showed that 
the actuation can affect the global flow field by modifying the evolution and interactions of the large-scale vortical structures. These modifications can lead to a Coanda-like deflection of the separating shear layer towards the surface, such that the layer vortices are advected downstream in close proximity to the surface as studied by Seifert et al. [17].

As discussed by Maldonado et al. [14], this approach was implemented to improve the aerodynamic performance of stalled airfoils and flaps [17] [18] [19]. Seifert et al. [17] and Wygnanski [20] stated that the flow control actuation is most effective when its frequency scales with the shedding frequency of the flow as measured by the 'time of flight' of the flow velocity over the chord length of the airfoil. When the separation domain scales with the characteristic length of an aerodynamic body, the actuation frequency (typically higher than the shedding frequency) can drive the shear layer in the near-field region or wake of the body It then becomes possible to control the shedding of coherent vortices in the shear layer when the flow begins to separate on an airfoil. This approach of coupling the actuation frequency to flow instabilities depends on the receptivity of the separating shear layer by the control input of secondary vortices in the form of passive vortex generators or active synthetic jets upstream of separation.

As reported by Maldonado et al. [14], another approach to control the flow is based on fluidic modification of aerodynamic lifting surfaces using embedded synthetic jets that are activated at high frequencies typically about an order of magnitude higher than the characteristic frequencies of the flow. This technique was implemented by Amitay \& Glezer [21]. It does not necessarily rely on coupling to global flow instabilities and thus can be applied over a broader range of flow conditions [22] [23]. Moreover, this approach can be applied to broader band control algorithms when unsteady actuation waveforms are used such as the pulse modulation technique [24] [25]. Earlier work [25] demonstrated that flow separation at high angles of attack can be mitigated by using synthetic jets that tailor the apparent surface curvature thereby modifying the distribution of the streamwise pressure gradient in advance of separation. For more information on flow control techniques for wind turbine blade applications, the reader is referred to a review book chapter by Maldonado [26].

\subsection{Motivation and Objectives}

The majority of the literature concerning wind turbine blade load and vibration reduction using blade distributed active flow control techniques deals with the deflection or deployment of aerodynamic control surfaces. However, these techniques can be overly mechanically complex to implement and/or not very efficient, i.e. the power needed to apply the control is of the same magnitude as the improvement. The objective of the present paper is to reduce a wind turbine blade model's structural vibration in a wind tunnel by efficiently controlling the blade's aerodynamic characteristics along its span for dynamic blade pitch maneuvers under the influence of dynamic stall conditions. This will be accomplished by spatially distributed arrays of mechanically simple, low power con- 
sumption synthetic jets near the leading edge for separation control, which in turn affects vibration due to suppression of the separating boundary layer.

The reduction of vibration helps increase the lifetime of the blades, and is an enabling technology that promotes the development of longer blades needed to capture more wind energy. The synthetic jet flow control system is integrated on these blades and is designed to work in parallel to conventional pitch actuators, but at a much higher bandwidth, making them effective in responding to gusts and other higher frequency atmospheric phenomena as mentioned earlier. This approach has the potential to reduce the size of conventional pitch actuators, and the distributed nature of the jets also has the ability to shed the excess lift from selected portions of the blade (this approach was examined by DeSalvo \& Glezer [27] on 2-D airfoils with promising results).

\section{Experimental Methodology}

A three-dimensional (3D) finite-span wind turbine blade model with an S809 airfoil was modeled in the Solidworks CAD package and rapid prototyped using a stereolithography technique with reinforced nylon material. The blade has a span of $40 \mathrm{~cm}$ and contains a root chord, $C_{r}=6.84 \mathrm{~cm}$ and a taper ratio, $C_{t} / C_{r}=$ 0.5 , where $C_{t}$ and $C_{r}$ are the tip and root chords respectively, and the mean aerodynamic chord is calculated as $5.32 \mathrm{~cm}$. The flow over the blade is expected to be three-dimensional in nature, particularly near the blade-tip region where spanwise flow is strongest. The blade contains no twist distribution and has an aspect ratio of 7.79, which represents an aspect ratio value closer to actual wind turbine blades. The modular design allows for independent access to four spanwise arrays of synthetic jets (20 jets total) located at a chordwise position of $x / c=$ 0.31 as shown on Figure 1. The synthetic jet piezo actuators were installed on cavities on the inside of the suction surface modules with rectangular orifices having a width of $0.5 \mathrm{~mm}$ and a length of $6.73 \mathrm{~mm}$.

The orifices are oriented horizontally and are co-linear as shown, creating a two-dimensional synthtic jet velocity profile with no preferred vortex rotation relative to its neighboring synthetic jet. This orifice oritentation matches that of a previous study by the author [28] in order to keep some synthetic jet variables the same and make a more approprate comparison of flow control effectiveness.

\subsection{Active Flow Control Technique}

The synthetic jet based flow control system consists of 20 spatially distributed synthetic jets driven with a $15 \mathrm{~mm}$ piezoelectric disk with an optimum driving frequency of $f_{\text {act }}=2350 \mathrm{~Hz}$ determined by measuring the synthetic jet velocity with a hot-wire anemometer. An electronic signal generator creates a sinusoidal waveform, which is imputed to a voltage amplifier utilized to power the actuators between 30 to 50 Volts. The characteristic shedding frequency of the flow over the model is $282 \mathrm{~Hz}$ (based on the "time of flight" over the mean aerodynamic chord) for a free stream velocity of $15 \mathrm{~m} / \mathrm{s}$. This velocity corresponds to a characteristic flow frequency that is about one order of magnitude lower than 


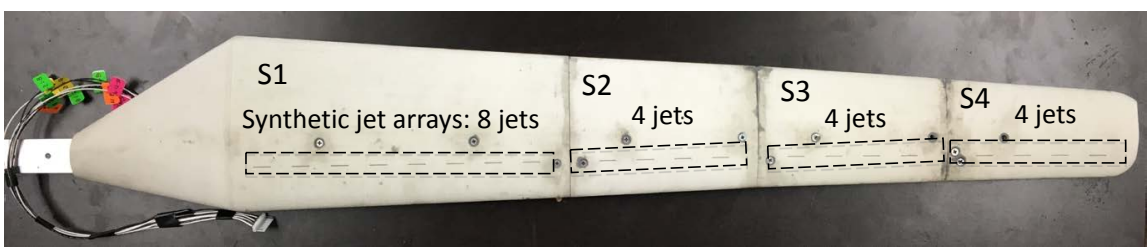

Figure 1. S809 wind turbine model with spanwise distributed synthetic jet actuators.

the actuation frequency, thereby affecting the coherent structures of the separating shear layer and causing some degree of flow reattachment.

According to Maldonado et al. [14], the strength of the synthetic jets (relative to the momentum of the external flow) is quantified using the momentum coefficient, $C_{\mu}$, defined as the following:

$$
C_{\mu}=\frac{n \bar{I}_{j}}{\frac{1}{2} \rho U_{\infty}^{2} A_{w}}
$$

where $U_{\infty}$ is the free stream velocity, $A_{w}$ is the blade area, $\rho$ is the density, and $n$ is the number of synthetic jets activated. $\bar{I}_{j}$ is the time-averaged synthetic jet momentum calculated during the blowing portion of the actuation cycle, defined as:

$$
\bar{I}_{j}=\frac{1}{\tau} \rho A_{s j} \int_{0}^{\tau} u_{j}^{2}(t) \mathrm{d} t
$$

where $\tau$ is the synthetic jet outstroke time, $A_{s j}$ is the area of the synthetic jet orifice, and $u_{j}(t)$ is the centerline velocity at the jet exit plane. The time-averaged jet moment is defined only for the outstroke part of the cycle and is normalized by the momentum of the external flow (for more details see Smith and Glezer [29]). For the experiments presented in this paper, the momentum coefficient $C_{\mu}$ was calculated as $2.30 \times 10^{-3}$ based on the activation of 20 synthetic jet actuators at an average jet exit-plane velocity of $12.5 \mathrm{~m} / \mathrm{s}$ and a free stream velocity of $15 \mathrm{~m} / \mathrm{s}$.

\subsection{Experimental Setup and Instrumentation}

The experiments were conducted in an open-return low-speed wind tunnel at UTSA. The wind tunnel has a test section manufactured from optically clear acrylic with a $46 \mathrm{~cm} \times 46 \mathrm{~cm}$ cross section area, and a length of $122 \mathrm{~cm}$. The tunnel is capable of generating a maximum air velocity of $30 \mathrm{~m} / \mathrm{s}$ with a $15 \mathrm{Hp}$ ducted motor creating a turbulence intensity level of less than $0.30 \%$ in the test section. The wind turbine blade model was mounted through a test section sidewall on a blade pitch actuator capable of generating static blade pitch angles of attack, as well as unsteady angle of attack motions by prescribing a dynamic pitch waveform. The computer controller was implemented with a National Instruments data acquisition system (NI USB-6341) and Labview software. The blade pitch actuator consists of a high-torque servo actuator with an internal potentiometer to track the angular position with an error of \pm 0.2 degree.

The blade model contained a pair of strain gauges to measure blade structural 
vibration by quantifying its unsteady tip deflection. The strain gauges were bonded on the upper and lower surface of the blade's aluminum spar near the root. This created a Wheatstone half-bridge, where each gauge produced an equal but opposite in magnitude strain, resulting in a higher resolution strain reading. The strain gauges were oriented in the spanwise direction to capture the bending or oscillating motion of the blade. The voltage output from the strain gauges due to spanwise bending was calibrated to the deflection at the tip. First, the voltage output was calibrated to the tip deflection by manually loading the blade with multiple weights at the tip and measuring its deflection. A second order curve of the tip deflection as a function of voltage was fitted to the data points and integrated into the Labview virtual instrument to generate time histories of the tip deflection. The strain gauge signals were processed through a signal conditioner and sampled with a 16-bit A/D acquisition board.

The global flow field over the suction surface of the blade was measured using a TSI stereoscopic particle image velocimetry (SPIV) system at a spanwise location of $y / b=0.53$ (about mid span) coinciding with the middle of a synthetic jet orifice. The SPIV system consists of a $1376 \times 1040$ pixel resolution thermo-electrically cooled 12-bit CCD camera, a pair of pulsed $120 \mathrm{~mJ} \mathrm{Nd:YAG} \mathrm{lasers,} \mathrm{and} \mathrm{a}$ programmable timing unit. The laser light sheet was emitted from below the wind tunnel section through a set of lenses enabling the positioning of the laser light sheet at the required spanwise location. A smoke generator (model Magnum 800 made by Martin Manufacturing PLC) was used together with standard smoke fluid to provide water-based droplets on the order of a few $\mu \mathrm{m}$ in diameter to serve as flow tracers. The smoke was introduced into the wind tunnel via the contraction section. The streamwise and cross-stream velocity components $(u, v)$ were computed from the cross-correlation of pairs of successive images with $50 \%$ overlap between the interrogation domains. For the time-averaged velocity vector fields, 250 image pairs were processed using an advanced multi-pass method where the initial and final correlation passes were $64 \times 64$ pixels and $16 \times 16$ pixels, respectively. The camera was mounted at a perpendicular distance of approximately $0.5 \mathrm{~m}$ to the laser light sheet, such that the distance between pixels is up to $62.5 \mu \mathrm{m}$. The maximum velocity $(30 \mathrm{~m} / \mathrm{s})$ corresponds to an average displacement of approximately 8 pixels with an error of \pm 0.1 pixels, which corresponds to a maximum error of $\pm 1.25 \%$ of the free-stream velocity $( \pm 0.38 \mathrm{~m} / \mathrm{s}$ at the maximum speed). A CAD schematic of the blade model is shown on Figure 2; illustrating the SPIV measurement domain and planes, coordinate system, and freestream velocity relative to the blade geometry and perpendicular to the synthetic jet orifices. .

The vibration control results below were performed at a free stream velocity of $15 \mathrm{~m} / \mathrm{s}$, resulting in a Reynolds number based on mean aerodynamic chord, $\operatorname{Re}_{\bar{c}}=5.28 \times 10^{4}$. The Reynolds number is defined as:

$$
\operatorname{Re}_{\bar{c}}=\frac{U_{\infty} \bar{c}}{v}
$$

where $U_{\infty}$ is the free stream velocity, $\bar{C}$ is the mean aerodynamic chord of the 


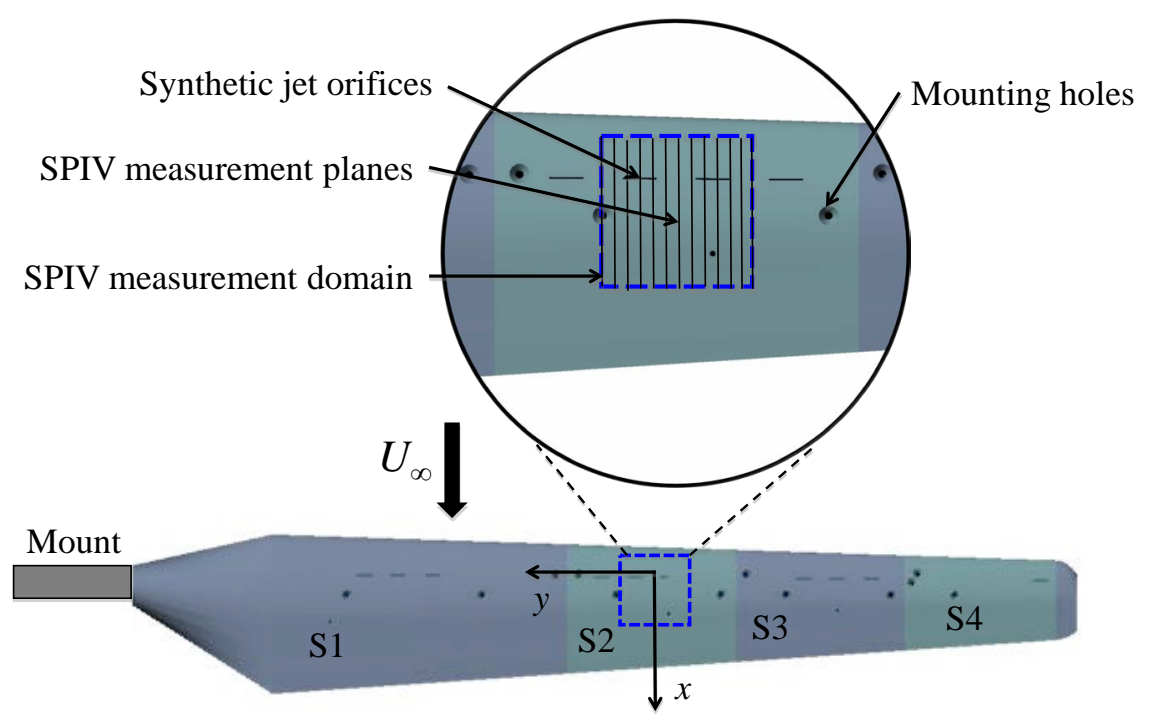

Figure 2. CAD schematic of wind turbine blade model and SPIV measurement details.

blade and $v$ is the kinematic viscosity of air at sea level temperature.

\section{Results and Discussion}

The effect of flow control on the structural vibration and mean flow of the wind turbine blade is investigated using strain gauge and SPIV techniques. In the first section, the effectiveness of the synthetic jets in reducing vibration under dynamic pitch conditions is explored. The global flow field measurements on section two reveal the impact of dynamic stall and the capacity for flow control to improve flow reattachment as a method to mitigate blade vibration. The flow field measurements were acquired for a sinusoidal pitch maneuver composed of multiple phase locked SPIV images along the sinusoidal pitch motion cycle.

\subsection{Active Vibration Control}

The mitigation of blade structural vibration is fundamentally correlated to the extent of flow re-attachment (for a stalled blade at post-stall angles of attack) as a result of synthetic jet actuation. The suppression or delay of the separating boundary layer via actuation prevents the formation of rotational flow next to the surface of the blade, which excites the blade at its fundamental structural resonance frequency [14]. As the angle of attack is further increased beyond stall, the rotational flow region thickens and increases in intensity. This is often accompanied by the shedding of a shear layer which passes over the region of recirculating flow and forms a wake, which leads to further and higher amplitude vibration of the blade.

When the blade undergoes dynamic pitching motion near the static stall angle of attack, the scenario above becomes more complicated as flow separation is now unsteady. Unsteady separation markedly varies the flow and aerodynamic properties of the blade such as the pressure gradient, lift, drag, and pitching moment around the separation point. These effects are part of a phenomenon 
known as dynamic stall. Dynamic stall occurs when the blade is subjected to any form of unsteady angle of attack motion which takes the effective angle of attack beyond its normal static stall angle. Occurrence of dynamic stall has been well documented in large scale wind turbines [30] [31] and thus there have been numerous studies, i.e. Leishman and Gupta [32] and Soltani et al. [33] to understand its effect on blade section aerodynamics and performance. Typically what is observed is when the angle of attack is increased, the onset of flow separation is delayed to an angle higher than the static stall angle, and, due to the shedding of a strong vortical structure from the leading edge which remains over the blade during a significant portion of the pitch up cycle, produces elevated values of lift. However, when the angle of attack decreases, flow re-attachment is found to be delayed to an angle of attack lower than the static stall angle. This leads to a significant hysteresis in the aerodynamics loads and thus vibration of the blade between the pitch up and down motion. In the experiments described below, synthetic jet flow control is used to mitigate blade vibration for unsteady separation conditions.

\subsubsection{Blade Structural Vibration}

The unsteady blade tip deflection time histories and mean tip deflection amplitudes were acquired for a series of dynamic pitch maneuvers that include linear "pitch up and down" as well as "sinusoidal pitch" at different reduced frequencies, defined as follows; $k=\omega c / 2 U_{\infty}$. Where, $\omega$ is the pitch rate of the blade in $\mathrm{rad} / \mathrm{s}$, and $c$ is the mean aerodynamic chord of the blade. Figure 3 presents the unsteady tip deflection time histories of the baseline case for a 'pitch up and down' maneuver at pitch rates of 2, 4, 6, $8 \mathrm{deg} / \mathrm{s}$ corresponding to reduced frequencies of $6.19 \times 10^{-5}, 1.24 \times 10^{-4}, 1.86 \times 10^{-4}$, and $2.48 \times 10^{-4}$ respectively.

The Reynolds number and synthetic jet momentum coefficient respectively was $\operatorname{Re}=5.28 \times 10^{4}$ and $C_{\mu}=2.30 \times 10^{-3}$, where all 20 synthetic jet actuators are activated together. The blade is pitched up from an initial angle of attack, $\alpha_{\mathrm{o}}=5^{\circ}$ to a peak, $\alpha_{\mathrm{p}}=20^{\circ}$, and immediately back down to $\alpha_{\mathrm{f}}=5^{\circ}$ according to the pitch function curve. The synthetic jets are activated at $\alpha=10^{\circ}$ on the pitch up motion, and turned off at $\alpha=10^{\circ}$ on the pitch down motion. As revealed by Figure 3(a) to Figure 3(d), the mean tip deflection increases fairly linearly at different rates (while keeping the tip deflection amplitude small) as the blade is pitched up until the onset of stall is reached. Note that as the angle of attack changes in the pitch up and down motion, the mean tip deflection increases and decreases according to the unsteady lift being produced by the blade. The blade tip deflection amplitude greatly increases when flow separation occurs near the static stall angle of attack of about $15^{\circ}$. Due to the effects of dynamic stall discussed earlier, however, flow separation and re-attachment (and thus the onset and halt of significant vibration) do not occur at a single static stall angle of attack; they occur at distinct angles of attack on the pitch up and down motion. The magnitude of the difference in angles becomes greater with increasing reduced frequency. For example, for $k=6.19 \times 10^{-5}$ (Figure $3(\mathrm{a})$ ) the angle of attack for the onset and 


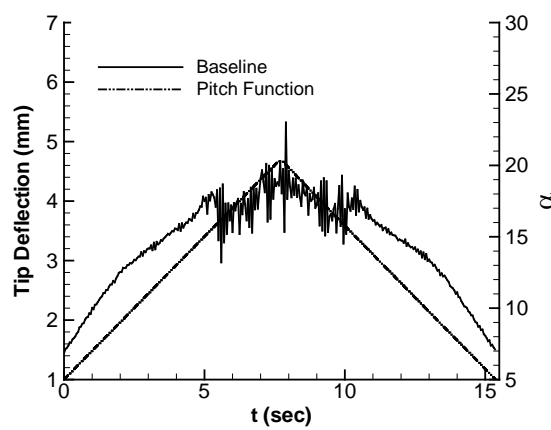

(a)

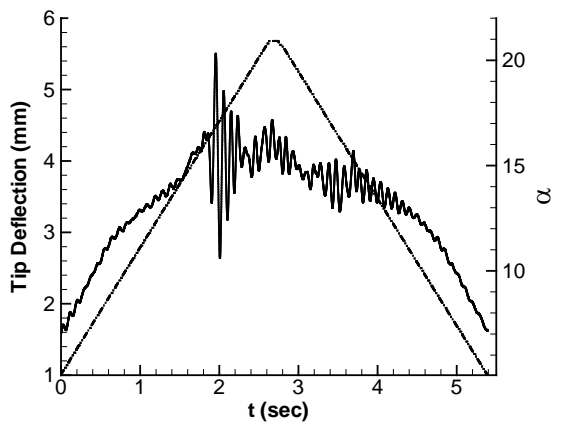

(c)

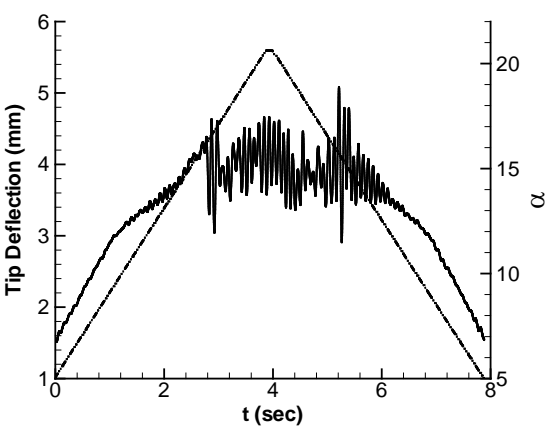

(b)

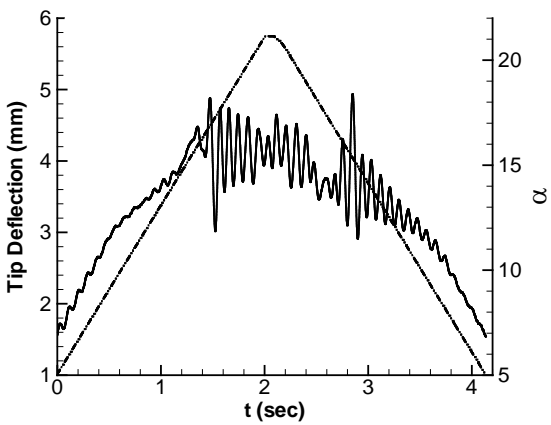

(d)

Figure 3. Tip deflection under dynamic blade "pitch up and down" at Re $=5.28 \times 10^{4}$ for reduced frequencies, of (a) $6.19 \times 10^{-5}$, (b) $1.24 \times 10^{-4}$, (c) $1.86 \times 10^{-4}$, and (d) $2.48 \times 10^{-4}$.

halt of considerable vibration is about $\alpha=18^{\circ}$ and $17^{\circ}$ on the pitch up and down motions respectively. However for $k=2.48 \times 10^{-4}$, (Figure 3(d)) vibration initiates and terminates at approximately $\alpha=17^{\circ}$ and $12^{\circ}$. Synthetic jet actuation (although not plotted together with the baseline cases to avoid overlapping of the data) offers some vibration reduction in regions particularly close to where vibration initiates and terminates, normally near the static stall angle of attack. However, it also amplifies vibration for other regions of the blade pitch cycle such as the peak (around $\alpha=20^{\circ}$ ) of the blade pitch motion.

Figure 4(a) to Figure 4(d) presents the tip deflection amplitude of the 'pitch up and down' maneuver for the baseline and flow control cases under the same experimental conditions as the prior case presented in Figure 3, however the mean tip deflection (prior to stall) is removed. Plotting the unsteady tip deflection amplitude places emphasis on the fluctuating component of the vibration (as opposed to the mean tip deflection and thus time averaged loads) which gives rise to cyclic loads which are a greater concern in maintaining the structural integrity of wind turbine blades. These results clearly demonstrate the precise locations during the pitch motion when sinusoidal actuation offers reduced tip deflection amplitude and when it intensifies vibration.

Another dynamic pitch maneuver studied was the "sinusoidal pitch", in which the blade was pitched according to a sinusoidal function about a mean angle of attack, $\alpha_{\mathrm{m}}$ and an angle of attack amplitude, $\alpha_{\mathrm{a}}$. Figure $5(\mathrm{a})$ to Figure 5(c) presents only the baseline results for the cases where the blade is prescribed with $\alpha_{\mathrm{m}}=17.5^{\circ}$, and $\alpha_{\mathrm{a}}=2.5^{\circ}$ for the same reduced frequencies, $k=$ (a) $6.19 \times 10^{-5}$, (b) $1.24 \times 10^{-4}$, (c) $1.86 \times 10^{-4}$ respectively. The Reynolds number and synthetic 


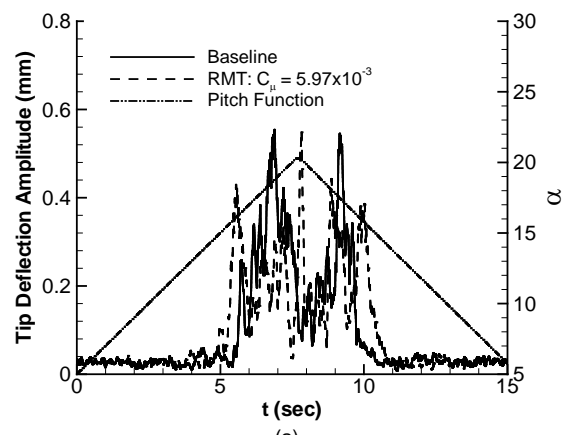

(a)

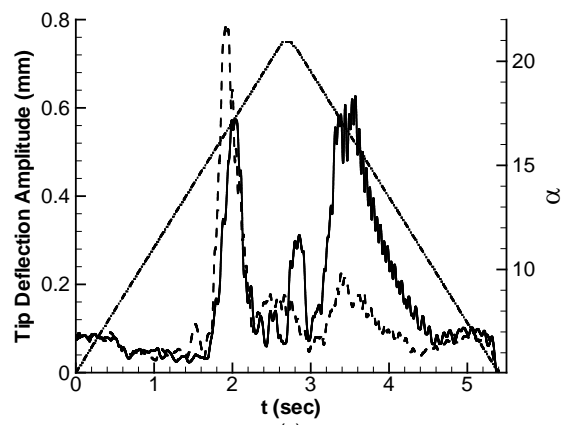

(c)

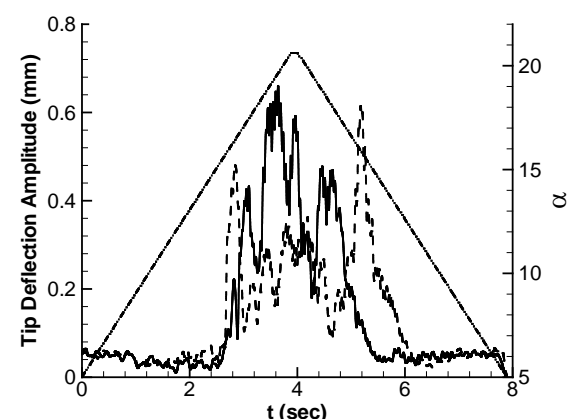

(b)

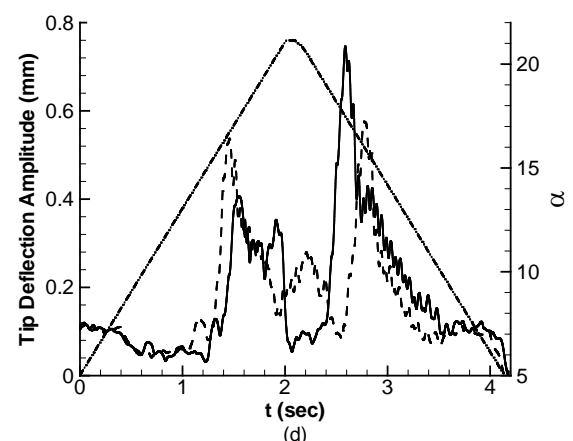

(d)

Figure 4. Tip deflection amplitude under dynamic blade "pitch up and down" at $\mathrm{Re}=$ $5.28 \times 10^{4}$ and $\mathrm{C}_{\mu}=2.30 \times 10^{-3}$ for reduced frequencies, $k$ of (a) $6.19 \times 10^{-5}$, (b) $1.24 \times$ $10^{-4}$, (c) $1.86 \times 10^{-4}$, and (d) $2.48 \times 10^{-4}$.

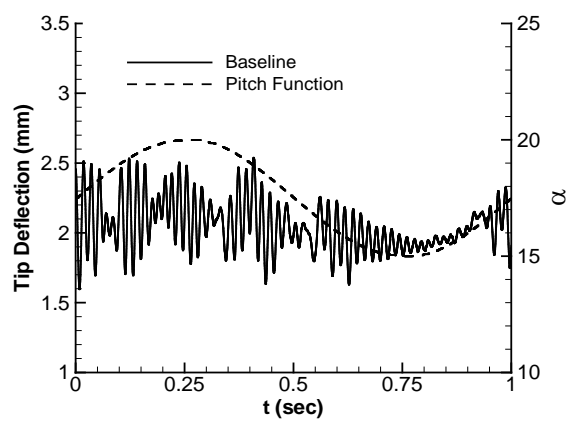

(a)

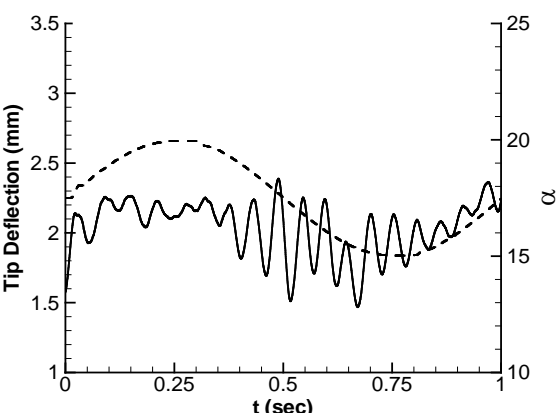

(b)

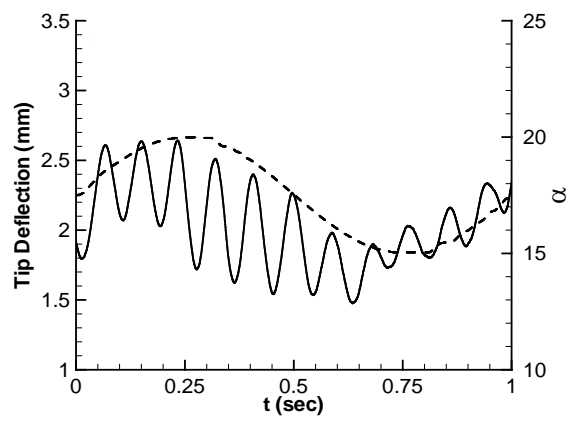

(c)

Figure 5. Tip deflection under dynamic blade "sinusoidal pitch" at $\mathrm{Re}=5.28 \times 10^{4}$ for reduced frequencies, $k$ of (a) $6.19 \times 10^{-5}$, (b) $1.24 \times 10^{-4}$, (c) $1.86 \times 10^{-4}$.

jet momentum coefficient values are held constant at $\mathrm{Re}=5.28 \times 10^{4}$ and $C_{\mu}=$ $2.30 \times 10^{-3}$. The synthetic jets were tuned on sufficiently before the beginning of the pitch maneuver at $\alpha_{\mathrm{m}}$ so as to neglect transient flow control effects. The hys- 
teresis effect between the up and down motions of the pitch cycle is apparent particularly on the second half of the cycle for the highest reduced frequencies on Figure 5(c). Here, for any given angle of attack, the magnitude of the tip deflections are larger for the pitch down motion (between $\alpha=17.5^{\circ}$ to $15^{\circ}$ ) compared to the pitch up motion (between $\alpha=15^{\circ}$ to $17.5^{\circ}$ ). These results are consistent to the previous "pitch up and down" results as well as to the model of dynamic stall, where again flow separation (and consequently increased vibration) is delayed to a higher angle of attack on the pitch up motion compared to the pitch down motion.

In order to gain some insight and more easily quantify structural vibration due to dynamic stall, the tip deflection amplitude for the cases above with flow control are computed in Figure 6(a) to Figure 6(c). The results indicate that actuation offers reduced tip deflections, a decrease of about $0.1 \mathrm{~mm}$ compared to the baseline for the second half of the cycle ( $t / T=0.5$ to 1 ), and a decrease of about $0.3 \mathrm{~mm}$ for the first third of the cycle ( $t / T=0$ to 0.35 ) for Figure 6(a) and (b) respectively. For the remaining portion of the pitch cycles, actuation produces the same level of vibration as the baseline cases. For the highest $k$ value in Figure 5(c), actuation improves performance for $t / T=0$ to 0.25 , where tip deflection amplitude decreases $0.2 \mathrm{~mm}$, and deteriorates performance for $t / T=0.25$ to 0.5 by increasing vibration amplitude by $0.15 \mathrm{~mm}$. There is also a general trend of decreasing vibration amplitude from the first set of pitch up and down motions in the first half of the pitch cycle, to the second set on the second half of

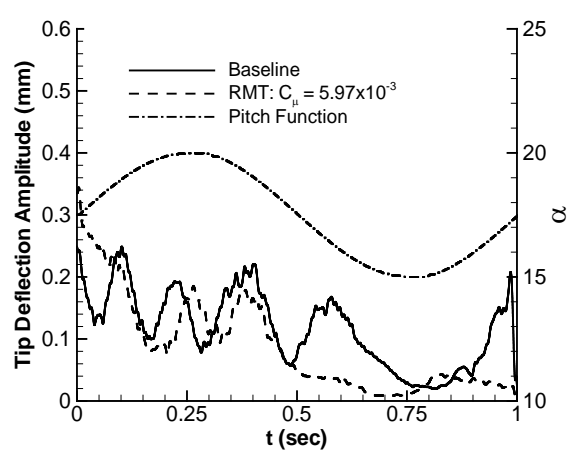

(a)

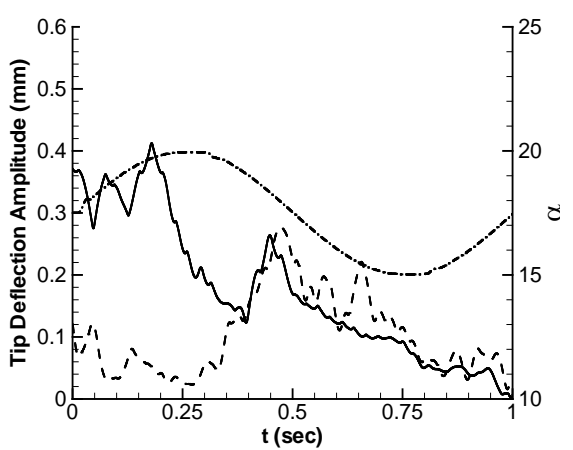

(b)

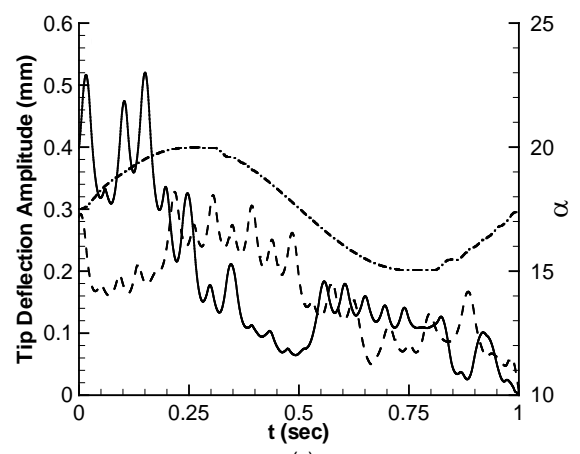

(c)

Figure 6. Tip deflection amplitude under dynamic blade "sinusoidal pitch" at $\operatorname{Re}=5.28 \times$ $10^{4}$ and $\mathrm{C}_{\mu}=2.30 \times 10^{-3}$ for reduced frequencies, $k$ of (a) $6.19 \times 10^{-5}$, (b) $1.24 \times 10^{-4}$, (c) $1.86 \times 10^{-4}$. 
the cycle. This suggests a hysteresis effect not only between the pitch up and down motions, but also between the first set of pitch up and down, and pitch down and up motions.

\subsubsection{Mean Global Flow Measurements}

To investigate the hysteresis effect in terms of the degree of flow separation on the suction surface of the blade, the global mean flow field was measured using phase-locked (to the pitch frequency) SPIV for the case presented in Figure 6(c) with a reduced frequency of $1.86 \times 10^{-4}$. This reduced frequency corresponds to a blade dynamic frequency of $1 \mathrm{~Hz}$. The average flow field was acquired with 200 image fields at each angle of attack throughout an entire "sinusoidal pitch" cycle. Data was taken starting at a mean angle of attack, $\alpha_{\mathrm{m}}=17.5^{\circ}$ with a $0.5^{\circ}$ increment and an angle of attack amplitude, $\alpha_{\mathrm{a}}=2.5^{\circ}$. This corresponds to a total of 20 angle of attack measurements. For the purposes of investigating the effect of dynamic stall on the global flow encountered in these experiments however, only the measurements of 3 angles during the pitch down and up motions of the "sinusoidal pitch" maneuver are discussed here. The angles of attack are $\alpha=17.5^{\circ}$, $16.5^{\circ}$, and $15.5^{\circ}$, which correspond to static angles of attack where the flow has stalled for 2-D flow on an S809 airfoil at low Reynolds numbers. The effect of flow control on the global flow during the pitch down motion are compared at $\alpha$ $=15.5^{\circ}, 16.5^{\circ}$, and $17.5^{\circ}$ for the baseline (Figure 7 (a) to Figure $7(\mathrm{c})$ ) and actuation using $C_{\mu}=2.30 \times 10^{-3}$ (Figure 7(d) to Figure 7(e)).

Notice the approximate location of the synthetic jet (at $\mathrm{x} / \mathrm{c}=0.31$ ) on the airfoil indicated by the black arrow on Figure 7(d) to Figure 7(f). The baseline and controlled flow at $\alpha=17.5^{\circ}$ and $16.5^{\circ}$ is completely separated and both show similar characteristics, where the center of the rotating flow structure lies just outside of the measurement field. However at $\alpha=15.5^{\circ}$, the center of the rotating flow structure lies closer to trailing edge surface for the controlled flow case, suggesting that actuation aids in partly re-attaching the flow. In addition, the size of the structure is significantly smaller than the baseline. A previous study by the author determined that the degree of flow separation and consequently the size of the rotating flow structures are correspondingly smaller for the baseline and actuation cases at static angles of attack [27] compared to the same angle when the blade is pitched down. This is consistent with the accepted phenomenon of dynamic stall as discussed earlier. Pitching the blade up however, results in the opposite effect; there is an improvement in the tendency for flow reattachment. In Figure 8, the blade is pitched up and the state of the flow is measured for the baseline (Figure 8(a) to Figure 8(c)) and actuation cases (Figure 8 (d) to Figure 8(f)) at $\alpha=15.5^{\circ}, 16.5^{\circ}$, and $17.5^{\circ}$ respectively. The rotating flow structure is now observed to be inside the measurement field for all angles of attack for the baseline and controlled flow.

The structures have traveled upstream towards the surface of the airfoil suggesting that the pitch up maneuver induces a favorable pressure gradient that alleviates the separated boundary layer. This is most likely due to the shedding of a 


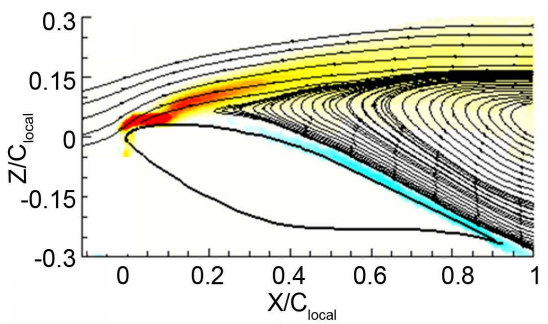

(a)

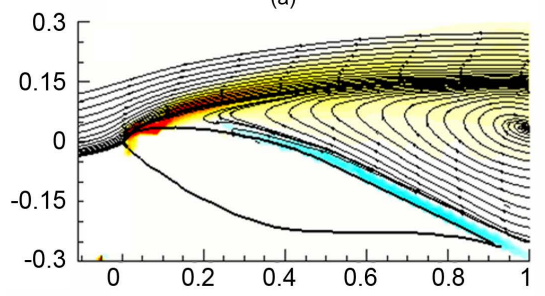

(c)

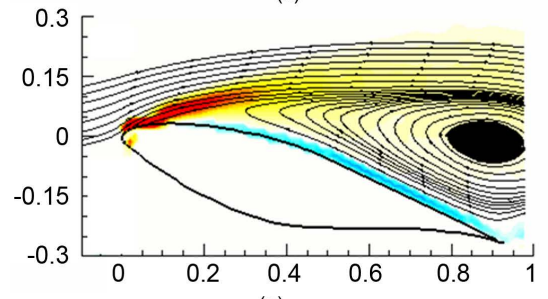

(e)

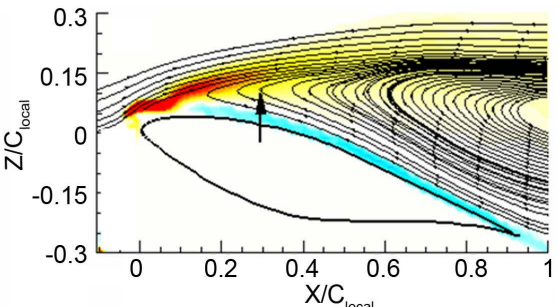

(b)

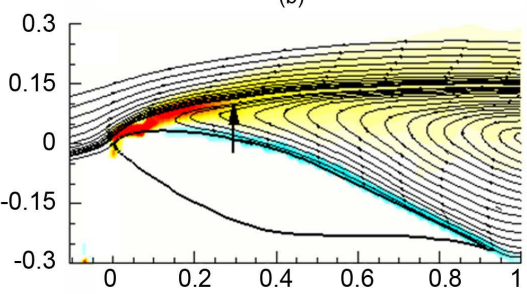

(d)

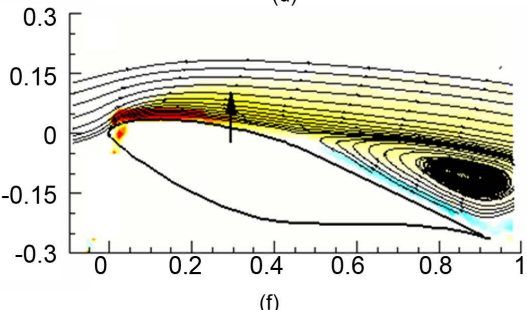

Figure 7. Global flow measurements at $\operatorname{Re}=5.28 \times 10^{4}, \mathrm{C}_{\mu}=2.30 \times 10^{-3}, \mathrm{y} / \mathrm{b}=0.53, \mathrm{k}$ $=1.86 \times 10^{-4}$ for baseline (a to c) and actuation ( $\mathrm{d}$ to $\mathrm{f}$ ) during the pitch down motion at $\alpha$ $=17.5^{\circ}, 16.5^{\circ}$, and $15.5^{\circ}$ respectively.
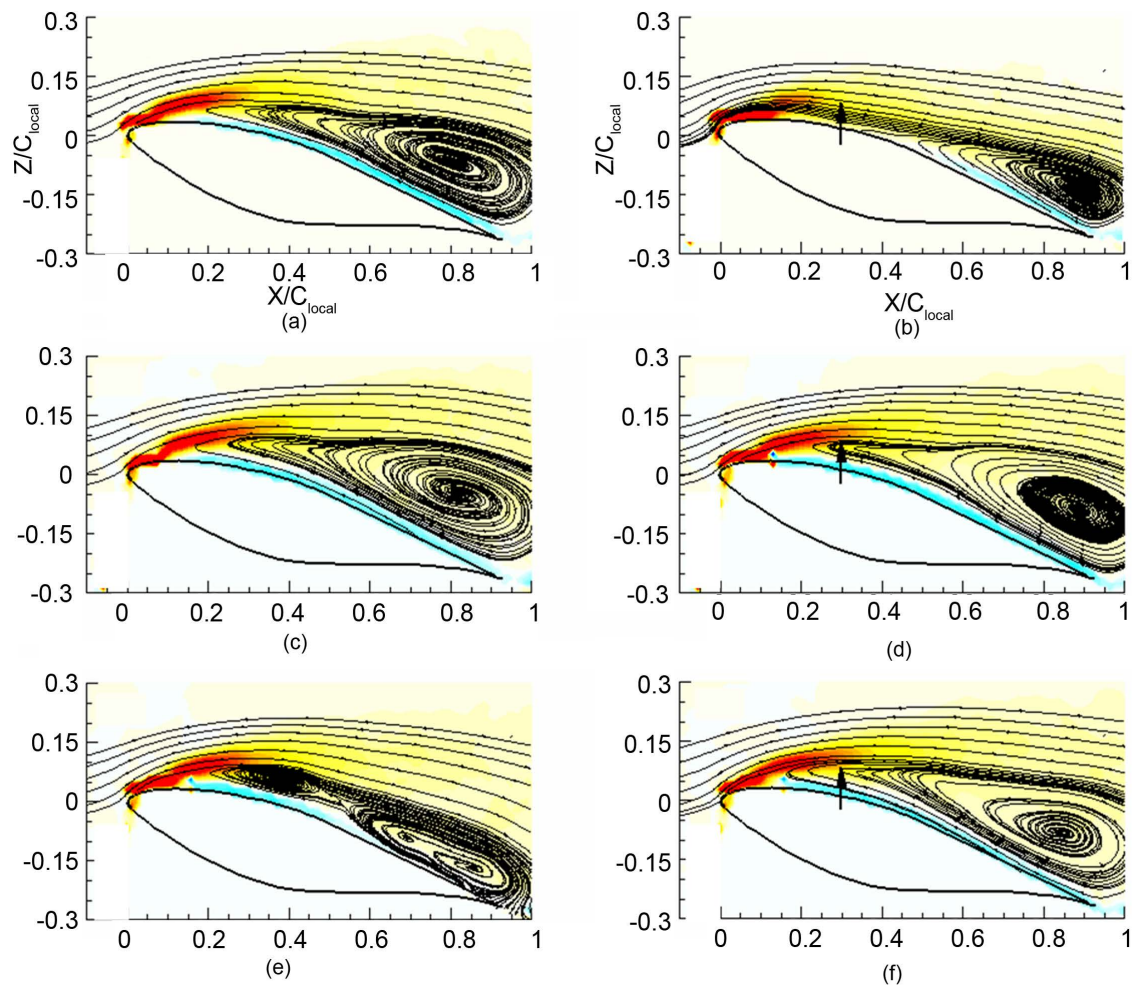

Figure 8. Global flow measurements at $\mathrm{Re}=5.28 \times 10^{4}, \mathrm{C}_{\mu}=2.30 \times 10^{-3}, \mathrm{y} / \mathrm{b}=0.53$, and $\mathrm{k}$ $=1.86 \times 10^{-4}$ for baseline (a) to (c) and actuation ( $\mathrm{d}$ to $\mathrm{f}$ ) during the pitch up motion at $\alpha$ $=15.5^{\circ}, 16.5^{\circ}$, and $17.5^{\circ}$ respectively. 
leading edge vortex that interacts with the boundary layer and developing shear layer. Moreover, a large laminar separation bubble is formed for the baseline case at $\alpha=17.5^{\circ}$ (Figure 8(c)) near the location of the synthetic jet that is not present and perhaps mitigated with flow control. The size of the primary recirculating structure is also smaller and contained closer to the suction surface compared to the forced case (Figure 8(f)), suggesting that flow control promotes separation during the pitch up maneuver at $\alpha=17.5^{\circ}$.

\section{Conclusion}

The implementation of a synthetic jet based flow control system for a low Reynolds number high aspect ratio wind turbine blade model was presented, and the reduction of structural vibration reduction was measured for dynamic blade pitch maneuvers representative of dynamic stall conditions. Flow control effectiveness was investigated in a wind tunnel at a free stream velocity of $15 \mathrm{~m} / \mathrm{s}$ corresponding to $\mathrm{Re}=5.28 \times 10^{4}$ for a range of dynamic angle of attack motions including linear "pitch up and down" and "sinusoidal pitch" maneuvers. The unsteady tip deflection and tip deflection amplitude of the blade under dynamic 'pitch up and down' and 'sinusoidal pitch' for low to moderate reduced frequencies, and $k$ was acquired revealing the portions of the pitch cycle where flow control offers vibration performance enhancement. Effects of dynamic stall were observed, especially for higher $k$ values where for a given post-stall angle of attack, and the blade tip deflections on the upward motion of the pitch maneuver were less than those for the downward motion. Global flow field measurements were also acquired using phase-locked SPIV at a spanwise location of $y / b=0.53$ for a range of angles during the pitch up and down phases of a 'sinusoidal pitch' maneuver. Improvements to flow reattachment and reduction of the recirculating flow structure were observed with flow control for both the pitch down and up motion at an angle of attack of $15.5^{\circ}$. Future work will concentrate on measuring the secondary vortex structures produced by the synthetic jets with SPIV in order to understand their interaction with the primary coherent structures in an unsteady separating boundary layer.

\section{References}

[1] Yen, D.T., Van Dam, C.P., Smith, R.L., and Collins, S.D. (2001) Active Load Control for Wind Turbine Blades Using MEM Translational Tabs. 39th AIAA/ASME, Reno, NV, 8-11 January 2001.

[2] Nakafuji, D.T.Y, Van Dam, C.P., Michel, J., and Morrison, P. (2002) Load Control for Wind Turbines-A Non-Traditional Microtab Approach. 40 th AIAA/ASME, Reno, NV, 8-11 January 2001.

[3] Baker, J.P., Standish, K.J. and Van Dam, C.P. (2005) Two-Dimensional Wind tunnel and Computational Investigation of a Microtab Modified S809 Airfoil. 43rd AIAA/ASME, Reno, NV, 10-13 January 2005.

[4] Chow, R. and Van Dam, C.P. (2007) Computational Investigations of Deploying Load Control Microtabs on a Wind Turbine Airfoil. 45th AIAA/ASME, Reno, NV, 8-11 January 2007. 
[5] Hulskamp, A.W., Beukers, A., Bersee, H., Van Wingerden, J.W. and Barlas, T.K. (2007) Design of a Wind Tunnel Scale Model of an Adaptive Wind Turbine Blade for Active Aerodynamic Load Control Experiments. 16th International Conference on Composite Materials, Tokyo.

[6] Joncas, S., Bergsma, O. and Beukers A. (2005) Power Regulation and Optimization of Offshore Wind Turbines Through Trailing Edge Flap Control. ASME Wind Energy Symposium, Reno, NV, 10-13 January, 2005.

https://doi.org/10.2514/6.2005-394

[7] Troldborg, N. (2005) Computational Study of the Riso-B1-18 Airfoil with a Hinged Flap Providing Variable Trailing Edge Geometry. Wind Engineering, 29, 89-113. https://doi.org/10.1260/0309524054797159

[8] Andersen, P.B., Guanaa, M., Bak, C. and Buhl, T. (2006) Load Alleviation on Wind Turbine Blades Using Variable Airfoil Geometry. European Wind Energy Conference, Athens, $8 \mathrm{p}$.

[9] Basualdo S. (2005) Load Alleviation on Wind Turbine Blades Using Variable Airfoil Geometry. Wind Engineering, 29, 169-182.

https://doi.org/10.1260/0309524054797122

[10] Bak, C., Gaunaa, M., Andersen, P.B., Buhl, T. and Hansen, P., Clemmensen, K., Moeller, R. (2007) Wind Tunnel Test on Wind Turbine Airfoil with Adaptive Trailing Edge Geometry. 45th AIAA Aerospace Sciences Meeting and Exhibit, Reno, NV, 8-11 January 2007. https://doi.org/10.2514/6.2007-1016

[11] Gaunaa, M. (2006) Unsteady 2D Potential-Flow Forces on a Thin Variable Geometry Airfoil Undergoing Arbitrary Motion. Technical Report Risø-R-1478(EN), Risø.

[12] Buhl, T., Gaunaa, M. and Bak, C. (2005) Potential of Load Reduction Using Airfoils with Variable Trailing Edge Geometry. Journal of Solar Energy Engineering, 127, 503-516. https://doi.org/10.1115/1.2037094

[13] Zayas, J.R., Van Dam, C.P., Chow, R., Baker, J.P. and Mayda, E.A. (2006) Active Aerodynamic Load Control for Wind Turbine Blades. European Wind Energy Conference, Athens, $8 \mathrm{p}$.

[14] Maldonado, V., Farnsworth, J., Gressick, W. and Amitay, M. (2009) Active Control of Flow Separation and Structural Vibrations of Wind Turbine Blades. Wind Energy, 13, 221-237. https://doi.org/10.1002/we.336

[15] Oster, D. and Wygnanski, I.J. (1982) The Forced Mixing Layer Between Parallel Streams. Journal of Fluid Mechanics, 123, 91-130. https://doi.org/10.1017/S0022112082002973

[16] Roberts, F.A. (1985) Effects of Periodic Disturbances on Structure of Mixing in Turbulent Shear Layers and Wakes. PhD Thesis, California Institute of Technology, Pasadena, CA.

[17] Seifert, A., Bachar, T., Koss, D., Shepshelovich, M. and Wygnanski, I. (1993) Oscillatory Blowing: A Tool to Delay Boundary-Layer Separation. AIAA Journal, 31, 2052-2060. https://doi.org/10.2514/3.49121

[18] Ahuja, K.H. and Burrin, R.H. (1984) Control of Flow Separation by Sound. 9th AIAA/NASA Aeroacoustics Conference, Williamsburg, VA, 15-17 October 1984.

[19] Neuberger, D. and Wygnanski, I. (1987) The Use of a Vibrating Ribbon to Delay Separation on Two-Dimensional Airfoils. Proceedings of Air Force Academy Workshop in Unsteady Separated Flows, Colorado Springs, CO, 28-30 July 1987.

[20] Wygnanski, I. (2000) Some Observations Affecting the Control of Separation by Periodic Excitation. AIAA Paper, 2314. https://doi.org/10.2514/6.2000-2314

[21] Amitay, M. and Glezer, A. (2002) Role of Actuation Frequency in Controlled Flow 
Reattachment over a Stalled Airfoil. AIAA Journal, 40, 209-216. https://doi.org/10.2514/2.1662

[22] Amitay, M., Horvath, M., Michaux, M. and Glezer, A. (2001) Virtual Aerodynamic Shape Modification at Low Angles of Attack Using Synthetic Jet Actuators. AIAA Paper, 2975. https://doi.org/10.2514/6.2001-2975

[23] Amitay, M. and Glezer, A. (2002) Controlled Transients of Flow Reattachment over Stalled Airfoils. The International Journal of Heat and Fluid Flow, 23, 690-699. https://doi.org/10.1016/S0142-727X(02)00165-0

[24] Amitay, M. and Glezer, A. (2006) Flow Transients Induced on a 2D Airfoil by Pulse-Modulation Actuation. Experiments in Fluids, 40, 329-331. https://doi.org/10.1007/s00348-005-0069-6

[25] Amitay, M., Smith, D.R., Kibens, V., Parekh, D.E. and Glezer, A. (2001) Modification of the Aerodynamics Characteristics of an Unconventional Airfoil Using Synthetic Jet Actuators. AIAA Journal, 39, 361-370. https://doi.org/10.2514/2.1323

[26] Maldonado, V. (2016) Active Flow Control of Wind Turbine Blades. In: Aissaoui, A.G. and Tahour, A., Eds., Wind Turbines-Design, Control and Applications, InTech, Rijeka, 303-324. https://doi.org/10.5772/63480

[27] Desalvo, M.E. and Glezer, A. (2007) Control of Airfoil Aerodynamic Performance Using Distributed Trapped Vorticity. 45th AIAA Aerospace Sciences Meeting and Exhibit, Reno, NV, 8-11 January 2007. https://doi.org/10.2514/6.2007-708

[28] Maldonado, V. Boucher, M., Ostman, R. and Amitay, M. (2009) Active Vibration Control of a Wind Turbine Blade Using Synthetic Jets. International Journal of Flow Control, 1, 227-237.

[29] Smith, B.L. and Glezer, A. (1998) The Formation and Evolution of Synthetic Jets. Physics of Fluids, 10, 2281-2297. https://doi.org/10.1063/1.869828

[30] Shipley, D.E., Miller, M.S. and Robinson, M.C. (1995) Dynamic Stall Occurrence on a Horizontal Axis Wind Turbine Blade. 14th ASME Wind Energy Symposium, Reno, NV, 9-12 January 1995.

[31] Robinson, M.C., Hand, M.M., Simms, D.A. and Schreck, S.J. (1999) Horizontal Axis Wind Turbine Aerodynamics: Three-Dimensional, Unsteady, and Separated Flow Influences. 3rd ASME/JSME Joint Fluids Engineering Conference, San Francisco, CA.

[32] Leishman, J.G. and Gupta, S. (2006) Dynamic Stall Modelling of the S809 Aerofoil and Comparison with Experiments. Wind Energy, 9, 521-547. https://doi.org/10.1002/we.200

[33] Soltani, M.R., Seddighi, M. and Marzabadi, F.R. (2008) Comparison of Pitching and Plunging Effects on the Surface Pressure Variation of a Wind Turbine Blade Section. Wind Energy, 12, 213-239. https://doi.org/10.1002/we.286 
Submit or recommend next manuscript to SCIRP and we will provide best service for you:

Accepting pre-submission inquiries through Email, Facebook, LinkedIn, Twitter, etc. A wide selection of journals (inclusive of 9 subjects, more than 200 journals)

Providing 24-hour high-quality service

User-friendly online submission system

Fair and swift peer-review system

Efficient typesetting and proofreading procedure

Display of the result of downloads and visits, as well as the number of cited articles Maximum dissemination of your research work

Submit your manuscript at: http://papersubmission.scirp.org/

Or contact ojfd@scirp.org 